\title{
La Vejiga Inestable en la Infancia
}

\author{
Dr. Jiggenio Rodrígucz S. $^{1}$; Dr. Jorge Holzer M. ${ }^{2}$; Dra. Ximena Triviño B. ${ }^{3}$; \\ Dr. Jorge Alvarcz L. ${ }^{3}$; $\mathbf{b}_{r}$. Carlos Saich A. ${ }^{1}$; Dr. Federico Puga $C^{1}$. \\ Urodinamic Studies in Patients with Urinary Infections, Enuresis \\ and or Urinary Incontinence.
}

\begin{abstract}
Fifty patienth from 4 to 13 years of age with recurrent urinary tract infection and both enuresis or urinary incontincnce were studied. All had nomal ncurologic findings. Intravenous urouraphy, uretrocistography, cistometry and in some of them sphincter electrowiography werc done. Nomal cistomelry accurred in $70 \%$, hipertonic responses in $20 \%$, hipotonic reactions in $4 \%$ and hiperreactive reflex responses in $6 \%$ of the patients of this serics. Correlations between age and vesical capicity witl the cistometric method are discussed. Different voiding disfunctions can be identified from urodinamic evaluation attending the pattern and intensity of biaddes contractions.

(Key words: Unstable bladder. Cistometry. Lrinary infection. Enuresis. Bladder incontinence).
\end{abstract}

En los últimos años se ha logrado un avance importante en técricas urodinámicas, útiles para conocer más a fondo la fisiología de la micción.

Ello ha permitido enfocar de manera más racional algunas enfermedades de la infancia como enuresis, incontinencia $\mathrm{e}$ infección urinaria recurrente, las que pueden presentarse por separado o simultáneamente ${ }^{1 \cdot 2-3-4 \cdot 5-6}$.

Es del mayor interés para e\} Podiatra-Nefrólogo continuar el estudio más allá de los exámenes radiológicos habituales --Pielografia y Uretrocistografía- en aquellos pacientes que presentan enuresis, incontinencia e infección urinaria recurrente, en algunos asociadas con encopresis, para pesquisar alteraciones funcionales, obtener un manejo adecuado y lograr resultados favorables ${ }^{1}$ $\cdot 2 \cdot 5 \cdot 7$

Se denomina vejiga inestable a ciertas alteraciones en el vaciamiento o llene de la vejiga, que no se asocian con trastornos neurológiços evidentes y sc manifestan habitualmente sólo como alteración funcional demostrable por estudios urodinámicos 5 .

La mayoría de las publicaciones enfocan el problema desde la perspectiva urodinámica relacionando esta con la forma clínica. Nos ha parecido interesante investigar desde la entidad clínica el trastorno funcional vesical, evidenciado por el estudio cistométrico, radiológico o ambos; determinar el grado de correlacióin entre edad,

1 Unidad de Nefrología, Hospital Luis Calvo Mackenna.

2 Instituto de Neurocirugía e Investigaciones Cerebrales.

3 Becado đe Peçiatría, Hospital Luis Calvo Mackenna. cistometría y capacidad vesical máxima y ade más, buscar si existe correlación entre infección urinaria recurrente y residuo vesical en el momento del estudio cistométrico.

Los estudios urodinámicos en general y en particular en los niños menores de 5 años, requie ren una interpretación cuidadosa por las difjcultades técnicas que presentan.

\section{MATERIALES Y METODOS}

Entre los años 1981 y 1983 se estudiaron 50 pacientes que consultaron en la Unidad de Nefro. logía del Hospital Luis Calvo Mackenna por infecciones urinarias recurrentes, enuresis, incontinencia o ambas. En todos se efectuaron pielografía de eliminación, uretrocistografía retrógrada, cistometria y en algunos electromiografía esfinteriana. Todos los nirnos eran neurológicamente normales.

La infección urinaria se consideró recurrente cuando el paciente sufrió 3 ó más episodios. Se defínió como enuresis a la emisión involuntaria nocturna de orina, por lo menos una vez por semana, en niños mayores de 5 años. Se conside. ró incontinencia urinaria a la emisión involunta. ria, diurna, de orina, por lo menos una vez al día, en mayores de 4 años de edad, en quienes se habian descartado causas extrauretales.

De los 50 pacientes, $43(86 \%)$ eran mujeres; de estas, 14 se presentaron con infección urinaria recurrente, 12 con las 3 entidades clínicas estudiadas, 9 con infección urinaria recurrente $y$ enuresis, 4 con enuresis, 3 con infección urinaria recurrente $e$ incontinencia y 1 con enuresis $e$ incontinencia. De los 7 varones, había 3 con enuresis, otros 3 con enuresis e incontinencia y 1 
con infección urinaria recurrente.

Las edades de los niños fluctuaron entre 4 años 5 meses y 13 años 6 meses, con promedio de 8,4 años.

El estudio urodinámico se efectuó mediante cistometría retrógrada. Esta consiste en el regis. tro de la presión intravesical durante el llene de la yejiga con solución de cloruro de sodio al $9 \%$ a través de una sonda uretral. La presión vesical se midió mediante un transductor de presión baja (statham) y se registró en un Poligrafo Multicanal Grass. Simultáneamente se midió la capacidad vesical máxima, definida como la suma del volumen emitido espontáneamente y cualquier residuo que quedase, al término de una micción precedida por un deseo urgente de orinar, des. pués de la infusión.

La electromiografía esfinteriana, que consiste en el registro de los potenciales bioeléctricos generados por la musculatura estriada del esfínter uretral externo, se realizó insertando un electrodo en el músculo en aquellos casos en que se comprobó residuo vesical elevado, es decir, mayor que el $5 \%$ de la capacidad vesical máxima, y por tanto sospechoso de disinergia entre el detrusor y el esfínter.

El reflejo anal se exploró por medio de la palpación de la contracción esfinteriana al pellizcar la unjón mucocutȧea. El reflejo bulbocavernoso se investigó estimulando fa zona del cuello vesical al traccionar la sonda Foley. Se realizó registro del tono anal.

En la cistometría se consideró que la vejiga era notmal cuando la amplitud del segmento de reflexia registrado (intensidad de la contracción del detrusor) corrèspondía a una presión equivalente a $45 \mathrm{~cm}$. de solución de cloruro de sodio al $9 \%$, hiperactiva si durante el examen ocurrieron contracciones vesicales no inhibidas y el segmento de reflexia variaba entre 45 y 50 centímetros de solución $\mathrm{Na} \mathrm{Cl} 9 \%$. Se definjó como vejiga hipertónica aquella cuyo segmento de reflexia superaba los 50 centímetros de solución y no presentaba contracciones del detrusor no inhibidas o involuntarias, e hipotónica aquella que la amplitud del segmento reflexia era inferior a 35 centímetros de solución. (Fig. 1)

La cistometría se realizó cuando había transcurrido por lo menos un mes sin infección del tracto urinario, especiaimente aquellos pacientes con infección urinaria recurrente.

\section{RESULTADOS}

Del total de 50 pacientes estudiados con cistometrías el $70 \%$ resultó normal, $20 \%$ tenía vejiga hipertónica, $4 \%$ vejiga hipotónica y $6 \%$ vejiga hiperactiva. (Fig. 1 ).

La relación entre la presentación clínica y el diagnóstico cistométrico se muestra en la Tabla 1.

La diferencia entre las capacidades medias de las vejigas hipertónicas, normales e hiperactivas no es significativa ( $p>0.05$ ), pero se encontra. ron diferencias significativas entre las capacidades vesicales normales $y$ las hipotónicas $(p<0.01)$. los valores promedios de las capacidades vesica. les según la alteración cistométrica se observan en la Tabla 2 .

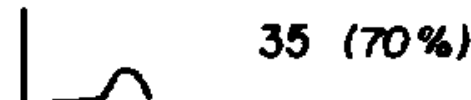

NORMAL
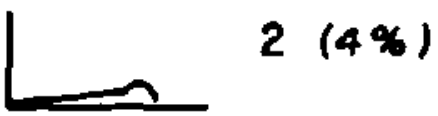

HIPOTONICA

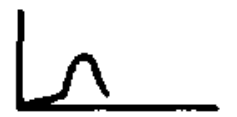

$10(20 \%)$

\section{HIPERTONICA}

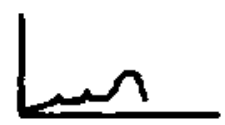

\section{$3(6 \%)$}

Figura 1

Registro de presión vesical (cistometría):

Tipos de curvas obscrvadas en 50 (100\%) pacien1es con antecedentes de infección urinaria y enuresis, incontinencia urinaria o ambas. 
Tabla 1.

Relación entre cistontetría y forma clínica.

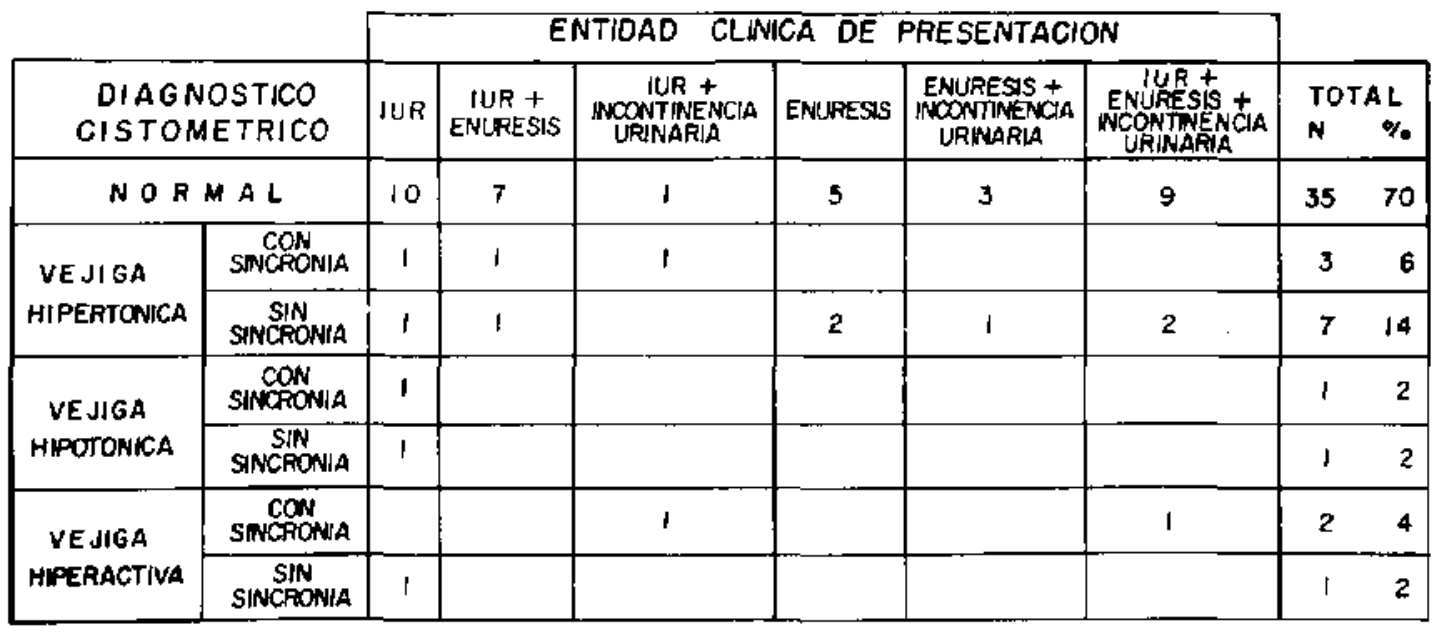

Tabla 2.

Capacidad vesical media según segmento de retlexia.

\begin{tabular}{ll}
\hline Ciasificación según & Capacidad mulia (m) \\
Segmento de relexia & $(1$ de $=58.6 \mathrm{ml})$ \\
\hline Vejiga notmal & $218\left(^{*}\right)$ \\
Vejiga hipertónica & 196 \\
Vejiga híperactiva & 201 \\
Vejiga hipotónica & $400(*)$ \\
\hline
\end{tabular}

$(* p<0,01)$.

De los 15 pacientes con alteraciones funcionales de la vejiga, 2 (13\%) tenían tambiér alteraciones radiológicas, ambos hidroureteronefrosis bilateral con reflujo vesicoureteral. Por otra parte, de los 35 pacientes cistométricamente nomales, 6 $(17,1 \%)$ presentaban alteraciones radiológicas importantes y algún grado de reflujo vesicoureteral.

En los pacientes con enuresis e infección urinaria recurrente, no se encontraron diferencias estadisticamente significativas $(p>0.1)$ entre la capacidad vesical máxima y el residuo post miccional.

\section{DISCUSION}

En nuestra casuística se encontró menor proporción de alteraciones funcionales de la vejiga en pacientes con infecciones urinarias recurrentcs, enuresis e incontinencia, que en otras experiencias ${ }^{1}$. Esto podría deberse a que los estudios de otros autores incluyen mediciones simultáneas de presiones vesicales y rectales, electromiografía esfinteriana, profilometría y flujometría en todos los pacjentes.
La clasificación de trastornos de la función vesical en vejigas hipertónicas, hiperactivas e hipotónicas, atendiendo a la capacidad vesical, parece ertada y contradictoria: nuestro estudio no mostró diferencias significativas para esta última de los grupos individualizados entre si y con sujetos normales, excepto en niños con vejigas hipotóricas, en los que dicha capacidad está francamente aumentada.

Por esta razón pensamos que para catalogar los diferentes comportamicntos y trastornos vesicales se deben considerar más bien la intensidad del segmento de reflexia y las contracciones no inhibidas del detrusor que la capacidad de la vejiga.

Es posible que el número de pacientes con cistometría normal sea también superior al de la literatura por considerar la intensidad del seg. mento de reflexia y no la capacidad vesical, como el método para definir estos trastomos.

Estamos de acuerdo con la literatura extranjera en cuanto a la distribución porcentual de los trastornos vesicales, excluyendo los catalogados como rornales. Es asi como en la infección urinaria recurrente el trastorno vesical predominante es la rejiga hipertónica y con menor frecuencia la hiperactiva y la hipotónica. Esta última disfunción vesical se presenta raramente en niños, sólo en infecciones urinarias recurentes, $y$ podríanos decir nunca en enuresis e incontinencia urinaria.

Por otra parte, la cistometría de niños con enuresis corresponde fundamentalmente a la forma hipertónica y en pocos casos a la hiperactiva.

El tipo de trastorno funcional vesical determina una terapéutica específica, que en la experjen. 
cia extranjera alcanza entre 60 y $70 \%$ de éxito. Es asi que los trastornos hipertónicos o hiperacti. vos del detrusor son tratados con drogas anticolinérgicas (Ej: Imipramina, bromuro de propantelina, hyoscina o cloruro de oxibutínico) y los hipotónicos con fármacos colinérgicos (Ej: Bromuro de neostigmina y cloruro de betanecol).

En el cuello vesical (esfínter interno) se puede actuar estimulándolo con drogas adrenérgicas como el sulfato de efedrina y fenilpropanolamina; inhiben la contracción esfinteriana las drogas simpaticolíticas como la fenoxibenzamina, metildopa y guanetidina ${ }^{8} \cdot 9$.

Nuestra experiencia terapéutica es escasa y será motivo de otra investigación.

\section{RESUMEN}

En cincuenta niños entre 4 y 13 affos de edad, neurológicamente normales, con infección urina. ria recurrente, y que además sufrían de enuresis, incontinencia 0 ambas, se efectuaron pielografías de elminación, urectrocistografías, cistometrías y en algunos de ellos electromiografía esfinteriana.

En el $70 \%$ de los casos la cistometría era normal, en $20 \%$ había vejiga hipertónica, en $4 \%$ ésta era hipotónica y en $6 \%$ hiperactiva.

Se discute la correlación entre edad y capacidad vesical en función de la cistometría.
Se definen las diferencias alteracionales vesicales desde el punto de vista cistométrico según la intensidad de la contracción del detrusor en el momento de la micción.

\section{REFERENCIAS}

${ }^{1}$ Bauer, S.B.i Retik. A.B.; Colodny, A.M.; et al: The unstable bladder of clithood. Symposium on pediatric urology. Urologic Clinics of North America. $2(7)$ : $231,1980$.

${ }^{2}$ Smey, P.; King, L.R.; Firlit, C.F.: Dysfunctional voiding is children secondary to internal sphincter dyssinergia: Treatment with phenoxybenzmine. Symposium on pediatric urology. Urologic Clinics of North America, 2 (7): 337, 1980.

${ }^{3}$ Firlit, C.F. Cook, $w . A$.: Voiding pattern abnormalities in chuldren. Urol. 10:25, 1977 .

4 Smey. P.: Firlit. C.F.; King, L.R.: Voiding pattem abnomalities in normal children: Results of pharmacologic inanipulation. J. Urol. 120: 594, 1978.

${ }^{5}$ Kaplon, G.W.; Brock, W.A.: Voiding dysfunction in children. Current problems in pediatrics. $8(\mathrm{X}): 4$, 1980.

${ }^{6}$ MC Loin, L.G.: Chilhood enuresis. Current problems in pediatrics. 8 (IX): $4,1979$.

7 Koff, S.A.: Effect of uninhibited bladder contractions on uti and reflux. Dialogues in pediatric urology. 12 (6): 6,1983 .

B Wein, A.J.: Urinary incontinence management. Dia. logues in pediatric urology. 3 (4): 3, 1981.

9 Raz, S.; Bradley, W'E.: Neuromuscular dysfunction of the lower urinary tract, Campbell's urology, fourth edition, vol, 2, pag. 1249, 1979.

10 Muether. S.R.: Development of urinary control in children. Some aspects of the cause and trcatment of primary enuresis. J.A.M.A. 172; 1256, 1960. 